\title{
Diagnóstico das Dificuldades nas disciplinas de Algoritmos I e de Algoritmos II de um Curso de Sistemas de Informação
}

\author{
Rodrigo Cardoso Oliveira, Soraia Silva Prietch \\ Universidade Federal de Rondonópolis - Av. dos Estudantes, 5055 - 78.736-901 - \\ Rondonópolis - MT - Brasil \\ rod.cardoso93@hotmail.com, soraia@ufr.edu.br
}

\begin{abstract}
This study presents the students' point of view about learning difficulties in Algorithms I and Algorithms II. The evident number of the courses' dropouts motivated the theme's choice. For this, we conducted an exploratory research by carrying out a questionnaire to students who had already attended the referred courses. Among the topics investigated were the following: content of the syllabus, programming languages, affinity with the Information Systems' area, dedication to extra-class studies, among others. The results of this research may be useful for restructuring computing undergraduate courses considering the students' opinions.
\end{abstract}

Resumo. Este estudo apresenta o ponto de vista de estudantes sobre as dificuldades na aprendizagem nas disciplinas de Algoritmos I e de Algoritmos II. A escolha do tema foi motivada pelo evidente número de desistências nas disciplinas. Para isso, pesquisa exploratória foi conduzida, tomando como base a aplicação de um questionário aos estudantes que já cursaram as referidas disciplinas. Dentre os temas investigados encontram-se: conteúdo das ementas, linguagens de programação, afinidade com a área de Sistemas de Informação, dedicação para estudos extraclasse, dentre outras. Os resultados desta pesquisa poderão ser úteis para a reestruturação de cursos de graduação em computação considerando a opinião dos estudantes.

\section{Introdução}

Disciplinas na área da programação são conteúdos obrigatórios em cursos de Sistemas de Informação e, com o estudo destas, o acadêmico constrói suas competências, adquirindo o conhecimento e as habilidades indispensáveis para projetar, modelar e construir programas e sistemas computacionais qualificados para a solução de problemas reais [Zorzo et al., 2017]. Relacionado ao tema, as dificuldades são inúmeras e tendem a crescer durante o curso, pois, se estas disciplinas oferecidas nos semestres iniciais se configuram em barreiras, situações decorrentes podem ser a evasão do curso [Hoed, (2016); Castro Junior et al. (2021)] ou ampliação das dificuldades em disciplinas posteriores que necessitam desses conhecimentos básicos para se aprofundar.

O objetivo geral desta pesquisa foi diagnosticar as dificuldades apontadas pelos estudantes no processo de ensino-aprendizagem das disciplinas de Algoritmos I e de Algoritmos II de um curso de Sistemas de Informação. A escolha do tema foi motivada pelo evidente número de desistências nas disciplinas, bem como pelo eminente início de discussões para a reestruturação do curso. 
O restante do artigo apresenta a Seção 2, com materiais e métodos utilizados para que o objetivo fosse atingido, a Seção 3, com referências sobre as dificuldades com disciplinas de algoritmos, a Seção 4, com dificuldades dos estudantes que cursaram Algoritmos I ou Algoritmos II em um curso de Sistemas de Informação do estado de Mato Grosso e, a Seção 5, com as considerações finais.

\section{Materiais e métodos}

A pesquisa aqui apresentada consistiu em um estudo com método indutivo que, conforme Marconi e Lakatos (2017), proporciona compreender o fenômeno observado por uma pesquisa com dados reais. Quanto à natureza, a pesquisa é aplicada que, de acordo com Gil (2010), constitui uma forma de aplicação prática, com a possibilidade de resolução de problemas. O tipo de pesquisa aplicada é a descritiva [Gil, 2010], a qual descreve os resultados segundo foram coletados, sem qualquer forma de influência do pesquisador, de modo a encontrar os problemas no caso analisado.

Os dados foram coletados com questionário online aplicado a vinte e dois estudantes graduandos que já cursaram ou estavam cursando as disciplinas de Algoritmos I e de Algoritmos II. O convite foi realizado via grupo de e-mail dos estudantes matriculados no curso. O questionário contou com dezesseis perguntas específicas para compreender o problema e conhecer potenciais estratégias de ensinoaprendizagem, sendo quatro questões abertas, cinco semifechadas e sete fechadas. A escolha pelo questionário ocorreu devido ao período de pandemia de Covid-19, sendo que Simone e Silva (2017) recomendam esta técnica para os casos de tentar abarcar uma quantidade maior de participantes e que inclua mais questões fechadas ou semifechadas. Para a análise dos dados, utilizou-se o método híbrido, que abrange métodos quantitativos e qualitativos simples [Marconi e Lakatos, 2017].

\section{Dificuldades com Disciplinas de Algoritmos}

A evasão nas disciplinas iniciais dos cursos de computação não é um fato novo. Segundo Gomes, Henriques e Mendes (2008), uma das razões para a acentuada desistência é a dificuldade encontrada pelos alunos com os conteúdos e habilidades necessárias nas disciplinas de programação. Silva e Trentin (2016) citam algumas dificuldades já identificadas em investigações prévias, tais como, a falta de competência na resolução de problemas, a inadequação dos métodos pedagógicos de ensino e também as linguagens de programação que possuem sintaxes adequadas para profissionais e não para iniciantes. Complementarmente Pereira Júnior (2006) e Rocha et al. (2010) apontam que existe também uma desmotivação para certas atividades, as quais estão relacionadas a uma carga de conceitos abstratos que intervêm em todo o conhecimento envolvido em projetar algoritmos, sendo que muitas vezes as características próprias das linguagens e ambiente de programação tendem a dificultar o processo.

Para Gomes, Henriques e Mendes (2008) existem diversos fatores que levam ao insucesso do estudante na compreensão da programação de computadores. Os autores (ibid.) reportam sobre a falta de interesse dos discentes pelo conteúdo proposto visto que exige persistência e prática, somado ao fato de não estudarem suficientemente o conteúdo para adquirir as competências necessárias dificulta ainda mais a aprendizagem. Além disso, para Silva et al. (2018), existem ainda outros obstáculos na compreensão do 
tema, tais como: a grande dificuldade na resolução de problemas, a falta de raciocínio matemático e lógico, pois o assunto é relacionado a estas áreas e a complexidade das linguagens de programação reais. De acordo com Lima Junior, Vieira e Vieira (2015), os conceitos, de Algoritmos para os acadêmicos do primeiro ano do curso, necessitam ser trabalhados de forma particular com base no ponto de vista da preocupação com a aprendizagem. Oferecer aprendizado nos fundamentos agregados aos ambientes de programação podem possibilitar melhor entendimento do conteúdo e realização de atividades didáticas de modo mais compreensivo.

\section{Dificuldades dos estudantes que cursaram Algoritmos I ou Algoritmos II em um curso de Sistemas de Informação do estado de Mato Grosso}

Para contextualizar quem foram os respondentes do questionário, dentre os vinte e dois voluntários, a média de idade foi de 23 anos, com faixa etária entre 19 e 41 anos, sendo $59,10 \%$ do gênero masculino e $40,90 \%$ do gênero feminino. Dentre eles, $36,06 \%$ já cumpriram a disciplina de Algoritmos I, 32,79\% de Algoritmos II e 31,15\% frequentaram a disciplina de laboratório de programação. Com relação a residir na mesma cidade da Universidade, 40,90\% dos alunos responderam que residiam na cidade desde que iniciaram a graduação, contudo sua residência era distante da instituição, $31,08 \%$ residiam na cidade e próximo a UFR, $18,20 \%$ comentaram que mudaram de cidade durante a graduação, 4,50\% residem em outra cidade e durante o período da graduação precisavam viajar todos os dias para estudar, e 4,50\% mencionaram que, no período de pandemia, com as aulas sendo realizadas remotamente ficou mais fácil por não necessitarem se deslocar para a Universidade. Além disso, como o curso de graduação em foco é ofertado no período noturno, descobrimos que $91,0 \%$ dos alunos respondentes trabalham em período integral.

Em linhas gerais, os depoimentos sobre a escolha do curso convergiram especialmente para uma categoria: afinidade/ familiaridade com a área. Tendo também uma resposta sobre a possibilidade de atuação no mercado de trabalho e a não disponibilidade do curso de Ciência da Computação na Universidade.

Sobre o conhecimento da matriz curricular ou do projeto pedagógico do curso, $50 \%$ dos alunos comentaram que buscaram este conhecimento ao ingressar no curso, 40,90\% mencionaram não realizaram esta busca, e 9,10\% escolheu a opção "outro" e comentou que ficou sabendo da grade por um colega que fazia o curso. A justificativa de alguns respondentes por não consultar foi que já tinham conhecimento sobre o curso ou já estavam realizando atividades profissionais na área, o que facilitou a escolha. Com relação ao conhecimento dos currículos de referências da Sociedade Brasileira de Computação (SBC), 95,50\% dos alunos disseram que não buscaram conhecer estes documentos e somente $4,50 \%$ deles tiveram o interesse de conhecê-los.

A respeito de ter participado de treinamento na área antes de escolherem ingressar no curso de Sistemas da Informação, 50,0\% disseram que nunca estudaram disciplinas de lógica de programação antes, 27,30\% comentaram que sim, com carga horária maior que $60 \mathrm{~h}$ em cursos de treinamentos externos, e 22,70\% participaram de treinamentos com carga horária menor que $60 \mathrm{~h}$. Dentre os que mencionaram que sim, incluíram cursos de: técnico em informática, C, Python ou Java. 
Com referência às dificuldades encontradas durante o período de estudo nas disciplinas de Algoritmos I e de Algoritmos II, 35,29\% apontaram dificuldades com raciocínio lógico, 26,47\% com a capacidade de abstração, $11,76 \%$ com a leitura e interpretação de textos, $8,82 \%$ com o conhecimento matemático, 5,88\% com a capacidade de generalização, 5,88\% não responderam nenhuma das opções, 2,95\% disseram que, de certa forma, falta de conhecimento e outros $2,95 \%$ relataram não ter dificuldades para o aprendizado do conteúdo.

Sobre as dificuldades com o conteúdo de Algoritmos I, observou-se que os estudantes relataram possuir maior dificuldade com matrizes $(23,80 \%), 19,04 \%$ com vetores, e 16,67\% com as estruturas de repetição. Com relação às dificuldades com o conteúdo de Algoritmos II, 26,31\% reportaram dificuldades com as funções com passagem de parâmetros, $18,42 \%$ com registros, seguindo com a mesma porcentagem de $18,42 \%$ o conteúdo de arquivos do tipo binário.

Quanto à linguagem de programação usada nas disciplinas como forma de exemplificação, os alunos apontaram as seguintes: na disciplina de Algoritmos I, Portugol e Pascal foram trabalhadas igualmente, seguidas pela linguagem $\mathrm{C}$ e depois pela linguagem Python, já na disciplina de Algoritmos II a linguagem de programação $\mathrm{C}$ foi a mais trabalhada no semestre, sendo realizadas atividades de Pascal também, mas com pouca frequência. Note que tais exemplos de linguagens foram citados por diferentes estudantes que cursaram as disciplinas em diferentes semestres letivos, portanto, isso não significa que quatro linguagens de programação foram usadas em um mesmo semestre.

Concernente aos estudantes que cursaram uma disciplina seguida da outra e as linguagens de programação usadas foram diferentes nas disciplinas de Algoritmos I e de Algoritmos II, foi solicitado que os alunos descrevessem os pontos positivos e negativos desta situação. A seguir são transcritos alguns dos depoimentos na íntegra:

- "O fato da linguagem ser diferente em Algoritmos I e II atrapalhou no meu aprendizado, creio que se fosse a mesma linguagem teria ajudado e facilito mais o aprendizado nas disciplinas".

- "O aluno se prende muito a linguagem e esquece que o principal é a lógica”.

- "Foi a mesma linguagem, e na minha opinião facilitou bastante o aprendizado em relação a Algoritmos II".

- "Positivos: sair do Pascal pro $C$ permite saber a estrutura de várias outras linguagens. Negativos: pode ser meio difícil aprender e se acostumar de início com a sintaxe de uma nova linguagem".

Sobre o tempo de dedicação para os estudos, na disciplina de Algoritmos I, nove dentre os respondentes relataram o tempo de até 4 horas semanais de atividades extraclasse, seis de até 2 horas, quatro mencionaram que estudavam apenas em sala de aula e, por fim, três relataram que estudavam mais de $8 \mathrm{~h}$ semanais. Na disciplina de Algoritmos II, onze relataram estudar até 4 horas semanais de atividades extraclasses, 6 alunos apontaram o tempo estimado em 2 horas semanais, seguido de dois que se dedicaram mais de 8 horas semanais. Outros dois relataram que nenhuma das opções se aplicavam, e um dos alunos entrevistados mencionou que estudava no horário de aula. 
Com respeito à busca pelos monitores em momentos de dúvidas sobre as disciplinas, 59,10\% informaram não buscar este tipo de auxílio, 31,80\% mencionaram que buscar suporte com os monitores do curso e, na opção outros, 9,10\% responderam, sendo que um dos alunos complementou que "pouquíssimas vezes procurou auxílio e que não podia ir nos horários dos monitores".

Ao questionar se os estudantes sabiam que o(a)s professore(a)s possuem horários de atendimento extraclasse em que podem solicitar agendamento de assistência diretamente com ele(a), 45,50\% dos alunos comentaram que não sabiam dessa possibilidade de atendimento, 40,90\%, disseram que sabiam mas nunca solicitaram o atendimento, $9,10 \%$ dos alunos respondentes solicitaram o atendimento e foram atendimentos e $4,50 \%$ responderam outros, sendo que um estudante informou que: "quando cursei eu não sabia, soube só depois e outros semestres a frente".

No que tange às estratégias de ensino-aprendizagem preferenciais, 26,68\% descreveram a preferência por vídeos no YouTube, 16,89\% pela colaboração em grupo, $11,69 \%$ por livros ou apostilas, 9,09\% por vídeos gravados pelo próprio professor da disciplina, $7,79 \%$ por slides de aula, 7,79\% por testes de mesa, $6,49 \%$ por desenhos de estruturas, 5,19\% pelo uso de ferramentas online para correção automática, 2,60\% selecionaram "outro", sendo que um estudante informou: "aulas do professor, documentação e slides voltados ao conteúdo".

Sobre os exercícios que os professores disponibilizam no Ambiente Virtual de Aprendizagem (AVA), 62,96\% disseram que tentam fazer o máximo que podem no tempo disponível que têm, $31,80 \%$ comentaram que somente fazem os exercícios se for para ganhar pontos no semestre e 18,52\% realizam os exercícios que for para contar como frequência em aula.

A última questão era opcional, aberta para que os respondentes incluíssem comentários e sugestões. Como resposta somente dois estudantes se pronunciaram com os seguintes comentários: (i) "Embora seja dificil aplicar novas estratégias em sala de aula, eu percebi que ainda existe um certo comodismo nos métodos de ensinamentos aplicados em sala de aula"; (ii) "Acredito que, mesmo que seja fácil pra alguns absolver conteúdo, muitos métodos aplicados em sala de aula ainda são ineficientes para alcançar a maioria. Buscar novas metodologias talvez possa ser um começo".

\section{Considerações finais}

Tivemos como objetivo com esta pesquisa diagnosticar as dificuldades apontadas pelos estudantes na aprendizagem nas disciplinas de Algoritmos I e de Algoritmos II de um curso de Sistemas da Informação de uma universidade de Mato Grosso. O estudo mostrou que são várias as dificuldades que os estudantes encontraram ao ingressar e tentar permanecer no curso.

Vinte e dois estudantes foram respondentes do questionário online, sendo que abarcou um público diverso em faixa etária e em gênero, além de estudantes que residem na cidade da Universidade e que residem no entorno. Não questionamos sobre a situação econômica familiar, mas a maioria dos respondentes trabalha durante o dia e frequenta as aulas no período noturno, o que reduz o tempo de dedicação extraclasse para estudos. 
Tanto o relato sobre afinidade com a área quanto a participação em cursos de informática e computação antes de ingressar no ensino superior apontam que os estudantes escolheram sua formação profissional embasada em decisões próprias, sem que fossem impostos por seus pais na falta de opção ou algo do estilo. A busca por conhecer a matriz curricular antes de ingressar no curso é regular e por currículos de referência da área é muito baixa, sendo estas perguntas feitas devido ao alto número de questionamentos de estudantes do motivo de certos conteúdos em disciplinas ou de disciplinas na matriz. Estratégias para que conheçam tais documentos podem ser pensadas para que estejam mais cientes sobre as razões da estruturação do curso.

$\mathrm{O}$ estímulo ao raciocínio lógico, à capacidade de abstração, e à leitura e interpretação de textos para compreensão de exercícios e de avaliações precisam ser efetuados como parte integrante das atividades em sala de aula, priorizando a interação, o diálogo e o senso crítico entre todos os envolvidos, ao invés da "transmissão" de conteúdos centrada no professor. Os três conteúdos mais críticos de Algoritmos I relatados pelos estudantes foram matrizes, vetores e estruturas de repetição, os quais estão todos relacionados, além de que vetores e matrizes são os conteúdos finais da disciplina, que talvez sejam prejudicados pela falta de tempo em aprofundá-los. Os conteúdos críticos da disciplina de Algoritmos II é praticamente a ementa completa: registros, funções e arquivos. Em funções com passagem de parâmetros e arquivos binários percebe-se maior dificuldade, supõe-se que devido ao conceito de ponteiros, que poderia ser melhor explorado nesta e em outras disciplinas.

Uma dificuldade a ser debatida no Núcleo Docente Estruturante (NDE) do curso é sobre a questão de mudança de linguagem de programação para uso em exemplificações nas disciplinas de algoritmos. A mudança é positiva para enriquecer o "cardápio" de opções dos estudantes em formação e pode ser negativa pela preocupação em aprender nova sintaxe em detrimento do conteúdo. Nota-se que os estudantes relatam realizar estudos extraclasse para ambas as disciplinas, o que é bastante positivo. No entanto, observou-se um número relativamente baixo da procura por monitores e da assistência do professor fora do horário de aula.

Com relação às estratégias de ensino-aprendizagem, um número expressivo de estudantes prefere assistir a vídeos, utilizar-se de livros, apostilas e apresentações de aulas, e também são favoráveis à promoção de colaboração em grupo. Haveria que verificar as estratégias utilizadas pelos professores, se atendem a esta demanda, uma vez que dois estudantes comentaram que existe "comodismo" e "ineficiência" no curso. Por fim, apesar da maioria relatar que tenta resolver a maior quantidade de exercícios disponibilizados no AVA, ainda existe um bom número de estudantes que somente os solucionam se houver algum tipo de estímulo, tais como, nota ou frequência.

Como continuidade deste estudo, recomenda-se realizar entrevistas, com questões abertas, para explorar em profundidade as respostas das questões fechadas e semifechadas presentes no questionário aplicado e também incluir outros tipos de indagações de interesse do curso.

\section{Referências}

Castro Junior, A. A. de, Cheung, L. M., Batista, E. J. S., Lima, A. C. de. (2021). Uma Análise Preliminar da Aplicação do Método 300 em Turmas de Algoritmos e 
Programação. In: Workshop Sobre Educação Em Computação (WEI), 29, Evento Online. Anais [...]. Porto Alegre: Sociedade Brasileira de Computação. p. 171-180. ISSN 2595-6175. DOI: https://doi.org/10.5753/wei.2021.15908.

Gil, A. C. (2010). Como elaborar projetos de pesquisa. 12. ed. São Paulo: Atlas.

Gomes, A., Henriques, J., Mendes, A. (2008). Uma proposta para ajudar alunos com dificuldades na aprendizagem inicial de programação de computadores. Educação, Formação \& Tecnologias. v. 1. n. 1. p. 93-103. Disponível em: https://eft.educom.pt/index.php/eft/article/view/23/16. Acesso em: 09 jan. 2021.

Hoed, R. M. (2016). Análise da evasão em cursos superiores: o caso da evasão em cursos superiores da área de Computação. Dissertação de Mestrado Profissional em Computação Aplicada, Departamento de Ciência da Computação, Universidade de Brasília. Disponível em t.ly/WxqM acesso em 06 Nov. 2021

Lima Junior, J. A. T., Vieira, C. E. C e Vieira, P. de P. (2015). Dificuldades no processo de aprendizagem de Algoritmos: uma análise dos resultados na disciplina de AL1 do Curso de Sistemas de Informação da FAETERJ - Campus Paracambi. Cadernos UniFOA, Edição 27. Disponível em: t.ly/vRJ1. Acesso em 12 out. 2021.

Marconi, M. A. Lakatos, E. M. (2017). Técnicas de Pesquisa. 8. ed. São Paulo: Saraiva.

Pereira Júnior, J. C. R. (2006). AVEP - Um ambiente virtual para apoio ao ensino de Algoritmos e programação. 124.p. Dissertação. Mestre em Engenharia de Produção. Centro de Ciências e Tecnologia. UENF, RJ.

Rocha, P. S., Ferreira, B., Monteiro, D., Nunes, D. da S. C., Góes, H. C. do N. (2010). Ensino e Aprendizagem de Programação: Análise da Aplicação de Proposta Metodológica Baseada no Sistema Personalizado de Ensino. Revista Renole. Novas Tecnologias na Educação. CINTED-UFRGS, v. 8. n. 3. dez. 2010.

Silva, B. S. da; Trentin, M. A. S. (2016). Dificuldades no ensino-aprendizagem de programação de computadores: contribuições para sua compreensão e resolução. V Simpósio Nacional de Ensino de Ciência e Tecnologia. II Semana Acadêmica da Licenciatura Interdisciplinar em Ciências Naturais. Disponível em: http://www.sinect.com.br/2016/down.php?id=3349\&q=1. Acesso em: 12 jan. 2021.

Silva, W. dos S., Lima, M. S., Raposo, J. C. dos S., Silva Junior, L. C. F. da. (2018). Levantamento sobre as dificuldades do discentes nas disciplinas de programação no curso técnico de informática. Diversitas Journal. v. 3. n. 3. p.761-770. set./dez. Santana do Ipanema-AL.

Barbosa, S. D. J. e Silva, B. S. da. (2017). Interação Humano-Computador. $2^{\mathrm{a}}$ ed., Editora Campus-Elsevier: Rio de Janeiro.

Zorzo, A. F., Nunes, D., Matos, E., Steinmacher, I., Leite, J., Araujo, R. M., Correira, R., Martins, S. (2017). Referenciais de Formação para os Cursos de Graduação em Computação, SBC. Disponível em: t.ly/kXgO. Acesso em: 8 fev. 2021. 